\title{
Fanconi anemia core complex-dependent HES1 mono-ubiquitination regulates its transcriptional activity
}

\author{
Cédric S. Tremblay ${ }^{1}$ (D), Feng Fei Huang ${ }^{2}$, Georges Lévesque ${ }^{3}$ and Madeleine Carreau ${ }^{4 *}$ (i)
}

\begin{abstract}
Objective: The Hairy Enhancer of Split 1 (HES1) is a transcriptional repressor that regulates cellular proliferation and differentiation during development. We previously found an interaction between HES1 and Fanconi anemia (FA) proteins. FA is a hematological and developmental disorder caused by mutations in more than 20 different genes. Eight FA gene products form a nuclear core complex containing E3 ligase activity required for mono-ubiquitination of FANCD2 and FANCl, both of which are FA proteins. Given that HES1 interacts with members of the FA core complex, the aim of this study was to determine whether HES1 is mono-ubiquitinated via the FA core complex.

Results: We show that HES1 is mono-ubiquitinated on a highly-conserved lysine residue that is located within a FA-like recognition motif. HES1 modification is dependent on a functional FA complex. Absence of HES1 monoubiquitination affects transcriptional repression of its own promoter. This study uncovers a novel post-translational modification of HES1 that regulates its transcriptional activity and suggests that ubiquitination of HES1 occurs in a FA core complex-dependent manner.
\end{abstract}

Keywords: Hairy-Enhancer of Split-1, HES1, Fanconi anemia, Ubiquitination, Mono-ubiquitination

\section{Introduction}

Transcriptional repressors of the Hairy-related basic helix-loop-helix (bHLH)-type family include Hairy and Enhancer of Split homologue 1 (HES1) [1-4]. We previously reported that HES1 interacts with Fanconi anemia (FA) proteins that compose the FA core complex, which modulates its transcriptional activity and self-repression $[5,6]$. Given the interaction of HES1 with FA core complex components and the FA core complex E3 ligase activity [5-10], we explored the possibility that HES1 might be a target of the FA core complex E3 ligase activity. Here, we present evidence that HES1 is mono-ubiquitinated in a FA protein-dependent manner. The HES1 protein sequence displays a putative FA-like recognition motif, which contains a conserved lysine residue.

\footnotetext{
*Correspondence: Madeleine.Carreau@crchul.ulaval.ca; Madeleine. Carreau@fmed.ulaval.ca

${ }^{4}$ Department of Pediatrics, Université Laval, CHUL, 2705 Boul. Laurier, RC-9800, Quebec, QC G1V 4G2, Canada

Full list of author information is available at the end of the article
}

Mutation of this lysine residue alters HES1 transcriptional activity. We propose that HES1 is a putative protein substrate of the FA core complex ubiquitin ligase activity, and that this post-translational modification regulates its transcriptional activity.

\section{Main text \\ Methods \\ Cells, plasmids and antibodies}

Cells used include: 293T (HEK293T; ATCC, Cedarlane Laboratories), HeLa (ATCC) COS-1 (ATCC, CRL-1650), PD430T (FA-A) fibroblast cell lines (gift) [5, 6]. Cells were grown at $37{ }^{\circ} \mathrm{C}$, in $5 \% \mathrm{CO}_{2}$ in DMEM media supplemented with $10 \%$ FCS. Plasmids used include: Myctagged ubiquitin (pCW7) or Myc-tagged ubiquitin K48R mutant (pCW8) coding vectors, HES1pro-Luc; pGL3p21 ${ }^{C I P 1 / W A F 1-2326 /+16}$ (p21pro-Luc) plasmids. Antibodies include: anti-FANCD2 (SantaCruz Biotechnologies clone FI-17 SC-20022 or Novus Biologicals NB100-182), rabbit polyclonal anti-HES1 (Chemicon AB5702 or SantaCruz 
Biotechnologies clone $\mathrm{H}-140, \mathrm{SC}-25392)$; monoclonal anti- $\beta$-tubulin (E7, Developmental Studies Hybridoma Bank); monoclonal anti-cMyc (SantaCruz Biotechnologies clone 9E10, SC-40), monoclonal anti-HA (Roche Diagnostics, clone 12CA5, \#11583816001), anti-ubiquitin (SantaCruz Biotechnologies clone N-19; SC-6085), Goat anti-mouse IgG-HRP conjugated (SantaCruz Biotechnologies, SC-2064), Goat anti-rabbit IgG-HRP (SantaCruz Biotechnologies, SC-2004). Transfections were performed using Lipofectamine 2000 (Invitrogen).

\section{Immunofluorescence, immunoprecipitation and Western blotting procedures}

Whole cell lysates were subjected to immunoblot or immunoprecipitation (IP) as described in [5, 6]. For IP, equal amounts of protein were incubated overnight at $4{ }^{\circ} \mathrm{C}$ with $2 \mu \mathrm{g}$ of antibodies followed by incubation with protein-G magnetic beads (Invitrogen). IP were resolved by sodium dodecyl sulfate-polyacrylamide gel electrophoresis (SDS-PAGE) and subjected to Western blotting with antibodies as indicated in each figure. Negative IP controls were performed using either mouse or rabbit serum. For immunofluorescence, HeLa cells were fixed in paraformaldehyde (2\%) and permeabilized with Triton $0.3 \%$ and labeled with primary antibodies followed with secondary antibodies as described previously in $[5,6]$ and in the figure legend. Randomization was performed during labeling. Labeled cells were visualized using the Nikon E800 fluorescent microscope equipped with a C1 confocal system (Nikon Canada) at $100 \times$ magnification.

\section{Luciferase assays}

COS-1 or FA-A fibroblast cells seeded in six-well plates were transfected with $0.38 \mu \mathrm{g}$ of the HES1pro-Luc vector, or $0.75 \mu \mathrm{g}$ of the p21pro-Luc (pGL3-p21 ${ }^{\text {CIP1/WAF1-2326/+16) }}$ promoter vectors together with $0.125 \mu \mathrm{g}$ of the pCMVLacZ control plasmid, as previously described [6]. The total amount of plasmid DNA was equalized between transfections using empty vectors. Cells were treated or untreated with mitomycin C $(120 \mathrm{ng} / \mathrm{ml})$ for $24 \mathrm{~h}$. Cell extracts were prepared $48 \mathrm{~h}$ following transfection and assayed for luciferase activity using the Luciferase assay system (Promega). Extracts were randomly distributed for analysis. Transfection efficiencies were normalized with pCMVLacZ using the $\beta$-galactosidase luminescence kit II (Clontech). Each experiment was performed at least twice in triplicates. Luciferase activity is expressed as relative luciferase units (RLU).

\section{Statistical analyses}

Data were expressed as means \pm standard errors of the means (SEMs). Statistical analyses were performed using the GraphPad Prism software (version 5.0b, GraphPad
Software Inc., San Diego, CA). Paired and unpaired twotailed Student's t tests were used to compare groups. Differences between means were evaluated using 2-way ANOVA with Bonferroni correction test. $\mathrm{p}$ values less than 0.05 were considered significant.

\section{Results \\ HES1 is mono-ubiquitinated in a FA core complex-dependent manner}

To determine whether HES1 as a FA complex partner is a substrate of the complex E3 ligase activity, 293T cells were transfected with Myc-ubiquitin (pCW7) or the dominant negative Myc-tagged ubiquitin mutant K48R (pCW8). The K48R mutant prevents the formation of multiubiquitin chains (polyubiquitination) through its lysine 48 residue, thus revealing mono-ubiquitinated forms [11]. Whole cell extracts were subjected to immunoprecipitation using anti-Myc antibodies (or mock IgG) and immunoblotted for HES1 and FANCD2 as a mono-ubiquitinated control protein. Both HES1 and FANCD2 were detected in Myc-ubiquitin immunoprecipitates from cells transfected with either wild-type ubiquitin (Fig. 1a lane 6) or the ubiquitin K48R mutant suggesting that HES1 is mono-ubiquitinated (Fig. 1a lane 7). We next examined HES1 ubiquitination in FA pathway-defective cells. HES1 was detected only in FA-A cells that expressed the wild-type ubiquitin protein but not the dominant negative ubiquitin K48R (Fig. 1a lanes 8 and 9, respectively) suggesting that mono-ubiquitination of HES1 requires a functional FA complex/pathway. Indeed, HES1 monoubiquitination (as well as FANCD2) was restored in FA-A cells after complementation with the FANCA gene as shown by immunoprecipitation using both anti-ubiquitin and anti-Myc antibodies (Fig. 1b). Immunoprecipitation using anti-Myc antibodies in FA-A mutant cells did not show any mono-ubiquitinated forms of endogenous HES1 or the long form of FANCD2 (Fig. 1c lane 6). However, endogenous HES1 mono-ubiquitination (as well as FANCD2) was restored in FA-A cells after complementation with the FANCA gene (Fig. 1c lane 7). Immunoprecipitation of HES1 using anti-Myc antibodies did not result from HES1 interaction with ubquitinated proteins such as FANCD2 since immunoprecipitates using antiHA antibodies showed the presence of HES1 but not FANCD2 (Fig. 1d, left panel). Also, immunoprecipitates using anti-ubiquitin antibodies confirmed that HES1 is ubiquitinated such as FANCD2 (Fig. 1d, right panel).

We searched HES1 conserved regions and lysine residues for a putative FA recognition sequence. We found that the lysine 109 residue, which is conserved in the HES1 sequence throughout evolution, is located within a putative FA recognition motif $\mathrm{V}(\mathrm{L} / \mathrm{I}) \mathrm{XK}$ (Fig. 1e). To determine whether the conserved lysine 109 is crucial 

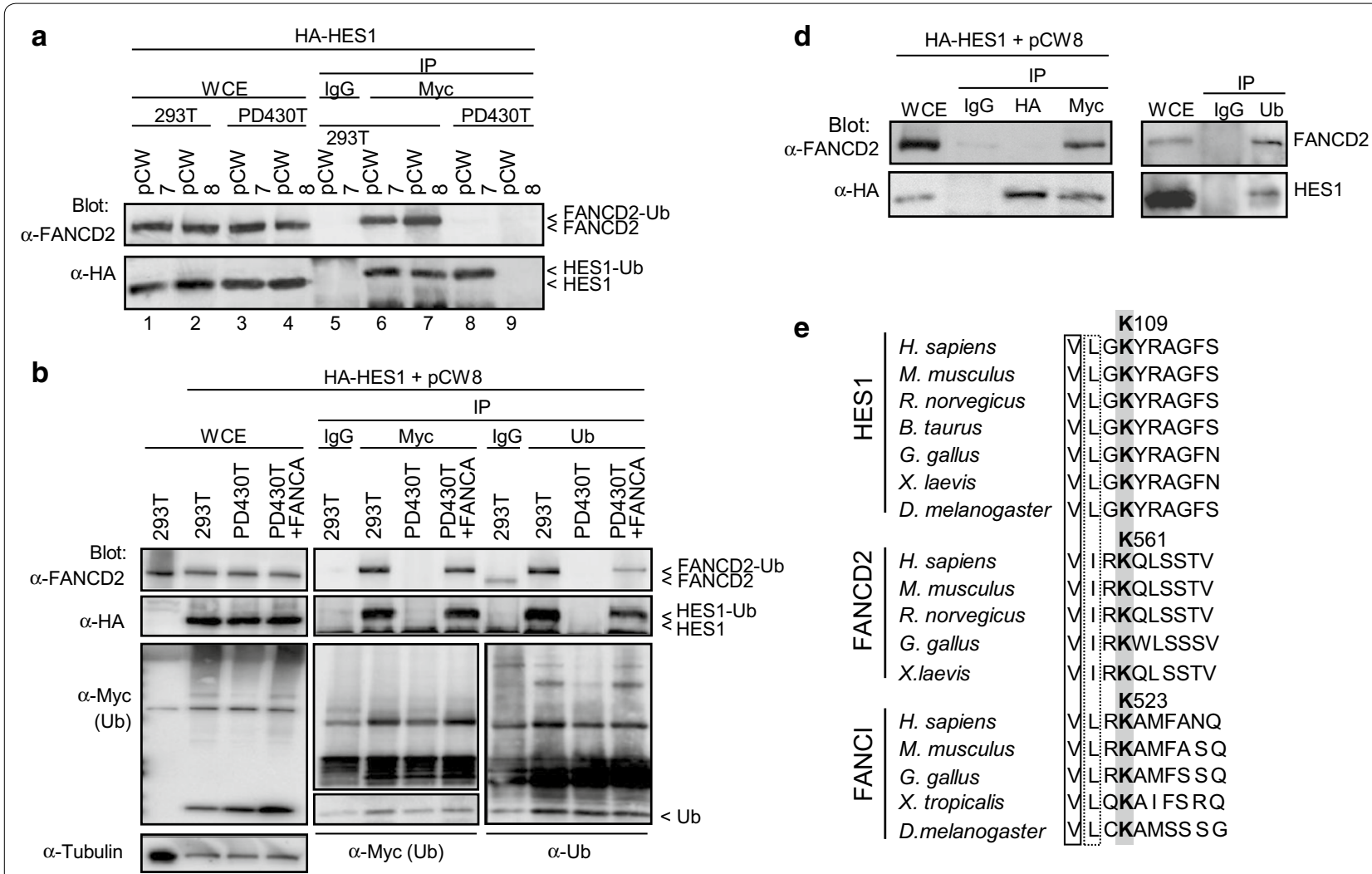

e

\begin{tabular}{|c|c|c|}
\hline & & \\
\hline & $s a$ & $\mathrm{~V} \mathrm{LG}$ \\
\hline & Ius & $\checkmark L G K Y$ \\
\hline & $n$ & $V L G$ \\
\hline & B. tal & $V L G$ \\
\hline & & VLG \\
\hline & $1 \mathrm{ag}$ & $V L G K$ \\
\hline & ster & $V L G K$ \\
\hline & & \\
\hline & ก. & VI:RK \\
\hline & M. $r$ & VIRK \\
\hline & R. nc & VIRKQLSSTV \\
\hline & G. gallus & VIRKWLSSSV \\
\hline & X.laev & VI:RK \\
\hline & & LRK \\
\hline & M. mus & VLRKAMFASQ \\
\hline & G. gallu & VLLRKAMFS SQ \\
\hline & & $\mathrm{V} / \mathrm{L} \mathrm{QK}$ \\
\hline & D.melanogas & VILCKA \\
\hline
\end{tabular}

C

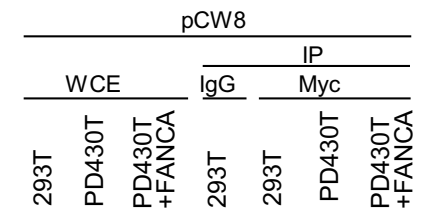
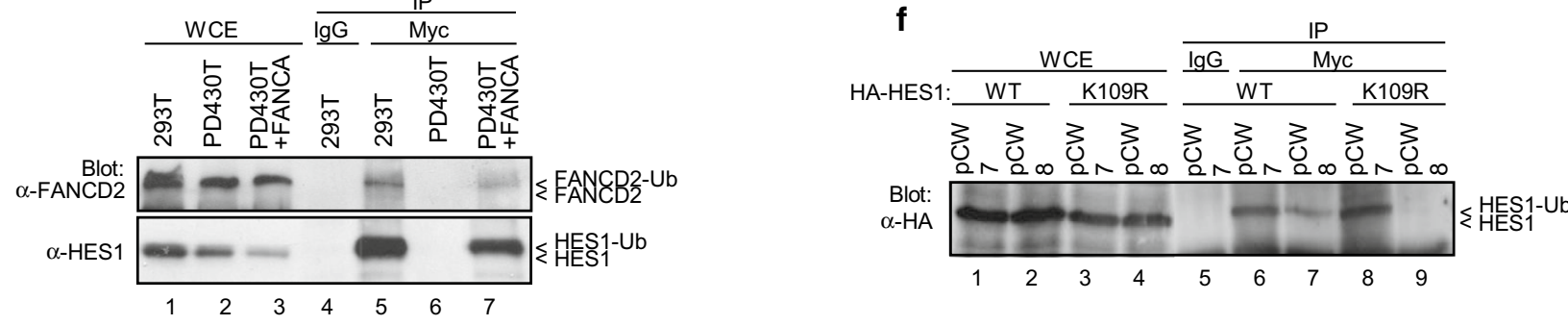

Fig. 1 HES1 mono-ubiquitination and its dependence on a functional FA complex. a In vivo ubiquitination of HES1. 293T and PD430T (FA-A) cells expressing HA-HES1 and Myc-tagged ubiquitin (pCW7) or the Myc-tagged ubiquitin K48R mutant (pCW8) were subjected to immunoprecipitation using anti-Myc antibodies or control lgG and were immunoblotted against HES1 and FANCD2. $\mathbf{b}$ Complementation of FA-A cells restores HES1 ubiquitination. PD430T (FA-A) cells and PD430T complemented with FANCA were transfected with HA-HES1 and the Myc-tagged ubiquitin K48R mutant (pCW8). Cell lysates were subjected to immunoprecipitation with anti-Myc or anti-ubiquitin antibodies. Western blotting was performed with the indicated antibodies. c Analysis of endogenous HES1 ubiquitination in FANCA mutant fibroblasts (PD430T). PD430T cells and PD430T complemented with FANCA were transfected with the Myc-tagged ubiquitin K48R mutant (pCW8) and were subjected to immunoprecipitation with anti-Myc antibodies. Western blotting was performed with the indicated antibodies. $\mathbf{d}$ Immunoprecipitation of HA-HESI using anti-Myc or anti-HA antibodies. 293T cells transfected with HA-HES1 and the Myc-tagged ubiquitin K48R mutant (pCW8) were subjected to immunoprecipitation with anti-HA or anti-Myc antibodies (left panel). Immunoprecipitation of endogenous proteins using anti-ubiquitin antibodies (right panel). Western blotting was performed with the indicated antibodies. e Alignment of the conserved region of HES1, FANCD2 and FANCI containing the putative FA recognition sequence. The peptidic sequence of HES1, FANCD2 and FANCI possess a V(I/L)XK sequence highly conserved through evolution. $\mathbf{f H E S} 1$ lysine 109 is crucial for HES1 mono-ubiquitination. 293T cells were transfected with HA-HES1 or HA-HES T $^{\text {109E }}$ COding vectors with the Myc-tagged ubiquitin K48R mutant (pCW8). Cell lysates were subjected to immunoprecipitation with anti-Myc antibodies or lgG control. Western blotting was performed with anti-HA antibodies. Antibody dilutions were used as follows: anti-HA at 1:5000; anti-HES1 at 1:1000; anti-FANCD2 at 1:1000; anti-tubulin at 1:10,000; anti-Myc at 1:500; anti-ubiquitin at 1:500; followed by secondary antibodies, anti-mouse, 1:10,000; anti-rabbit, 1:20,000 
for HES1 mono-ubiquitination, a K109E mutant form of HES1 was generated. The mono-ubiquitinated form of HES1 was immunoprecipitated only in cells expressing the wild-type HES1 but not the K109E mutant (Fig. 1f) suggesting that this residue is crucial for HES1 monoubiquitination. Together these results suggest that HES1 is monoubiquitinated in a FA core complex-dependent manner.

\section{Mono-ubiquitination of HES 1 has no effect on its cellular localization}

We previously showed that HES1 is partially localized to FANCD2-containing foci in MMC-treated cells [5]. Given that MMC-induced nuclear foci contain the mono-ubiquitinated form of FANCD2, we tested whether HES1 mono-ubiquitination is required for localization to MMC-induced foci. Results show that both wild-type and K109 mutant forms of HES1 are located to the nucleus (Fig. 2a, right panels). In addition, MMC treatment of HES1-transfected cells reveals partial localization of wild-type and mutant HES1 to FANCD2-conatining nuclear foci (Fig. 2a, left panels). These results indicate that mono-ubiquitination has no impact on HES1 cellular localization in non-treated and MMC-treated cells.

Given that HES1 binds its own promoter to repress its own transcription $[4,12]$, we tested whether HES1 post-translational modification regulates this auto-negative feedback mechanism. Using the HES1 promoter region to drive the luciferase gene (HES1pro-Luc), we tested the transcriptional repression capacity of HES1 K109-mutant. Results show that $\mathrm{HES}^{\mathrm{K} 109 \mathrm{E}}$ has reduced transcriptional repression capacity compared to the wildtype HES1 protein (Fig. 2b). Considering that the FA core complex ubiquitin ligase activity is known to modify its substrates in response to DNA damage [7-9], we tested whether HES1 or $\mathrm{HES} 1^{\mathrm{K} 109 \mathrm{E}}$ transcriptional repression is modulated following DNA damage. Results show that MMC treatment induced activation of the HES1 promoter as compared to untreated cells (Fig. 2b). Surprisingly, HES1 failed to repress its own promoter following MMC treatment as shown by a two-fold HES1 promoter activation compared to control cells. In addition, the HES $1^{\text {K109E }}$ further increased HES1 promoter activation compared to control cells and untreated cells. These results suggest the MMC treatment promotes HES1 promoter activation by preventing HES1-mediated repression. These results also suggest that the HES1 lysine 109 acts as a regulation site for its repressor function.

\section{Mono-ubiquitination of HES1 is required for transcriptional repression of its own promoter}

To determine whether FA complex-mediated regulation of HES1 occurs via mono-ubiquitination of HES1 lysine
109, we evaluated HES1 gene activation in FA-A cells in the presence of HES1 or $\mathrm{HES} 1^{\mathrm{K} 109 \mathrm{E}}$. Results show that HES1 transcriptional regulation is impaired in FA-A cells and in FANCE-transfected FA-A cells but restored in FANCA-corrected cells, thus confirming previous findings (Fig. 3a). We also confirmed that HES1-mediated repression of its own promoter is attenuated in FANCAcorrected cells compared to control FA-A cells and FANCE-transfected FA-A cells (Fig. 3a). However, we found that $\mathrm{HES} 1^{\mathrm{K} 109 \mathrm{E}}$ failed to repress its own promoter in FA-A cells despite FANCA-gene correction (Fig. 3a) or overexpression of FA core complex components (Fig. 3b).

Given that expression of the Notch1 intracellular domain (NICD), which is the active form of Notch1, has been shown to activate HES genes, including HES1, and that FA complex components enhanced NICD-mediated activation of the HES1 promoter, we tested the effect of $\mathrm{HES} 1^{\mathrm{K} 109 \mathrm{E}}$ on NICD-mediated HES1 activation. We found that, like HES1, HES1 ${ }^{\mathrm{K} 109 \mathrm{E}}$ repressed its own promoter independently of NICD-mediated activation of HES1 (Fig. 3c). Surprisingly, FA core-complex components failed to enhance NICD-mediated activation of HES1 in the presence of HES1 ${ }^{\mathrm{K} 109 \mathrm{E}}$ (Fig. 3c). Together, these results suggest that HES1 mono-ubiquitination is required for repression of its own promoter and is required for transcription of HES1 following NICDmediated activation in a FA core complex dependent manner.

We next determined whether the FA core complex modulates HES1-mediated repression of $p 21^{C I P 1 / W A F 1}$ via mono-ubiquitination of HES1. We found that HES1 ${ }^{\mathrm{K} 109 \mathrm{E}}$ was able to repress the $p 21^{C I P 1 / W A F 1}$ promoter more efficiently than the wild-type HES1 protein (Fig. 3d). Overexpression of FA core complex components further increased $\mathrm{HES} 1^{\mathrm{K} 109 \mathrm{E}}$-mediated repression of the $p 21^{\mathrm{CIP} 1 /}$ ${ }^{W A F 1}$ promoter. In addition, we found that $\mathrm{HES} 11^{\mathrm{K} 109 \mathrm{E}}$ like wild-type HES1, repressed the $p 21^{C I P 1 / W A F 1}$ promoter in FA-mutant cells, whereas complementation of FA-A cells with FANCA but not FANCE further increased this repression (Fig. 3e). These results suggest that monoubiquitination of HES1 is not required for transcriptional repression of the target gene $p 21^{\text {CIP1/WAF1 }}$.

\section{Discussion}

The HES1 protein is highly regulated through an oscillation-type mechanism, which occurs in part by an autonegative feedback mechanism at the transcription levels and rapid ubiquitination-dependent proteosomal degradation $[13,14]$. Recently the RBX1-CUL1-SKP1 ubiquitin ligase complex was shown to regulate HES1 degradation via poly-ubiquitination on several lysine residues [15]. In this publication, Chen et al. [15] revealed that HES1 polyubiquitination occurs at the conserved lysine residue 


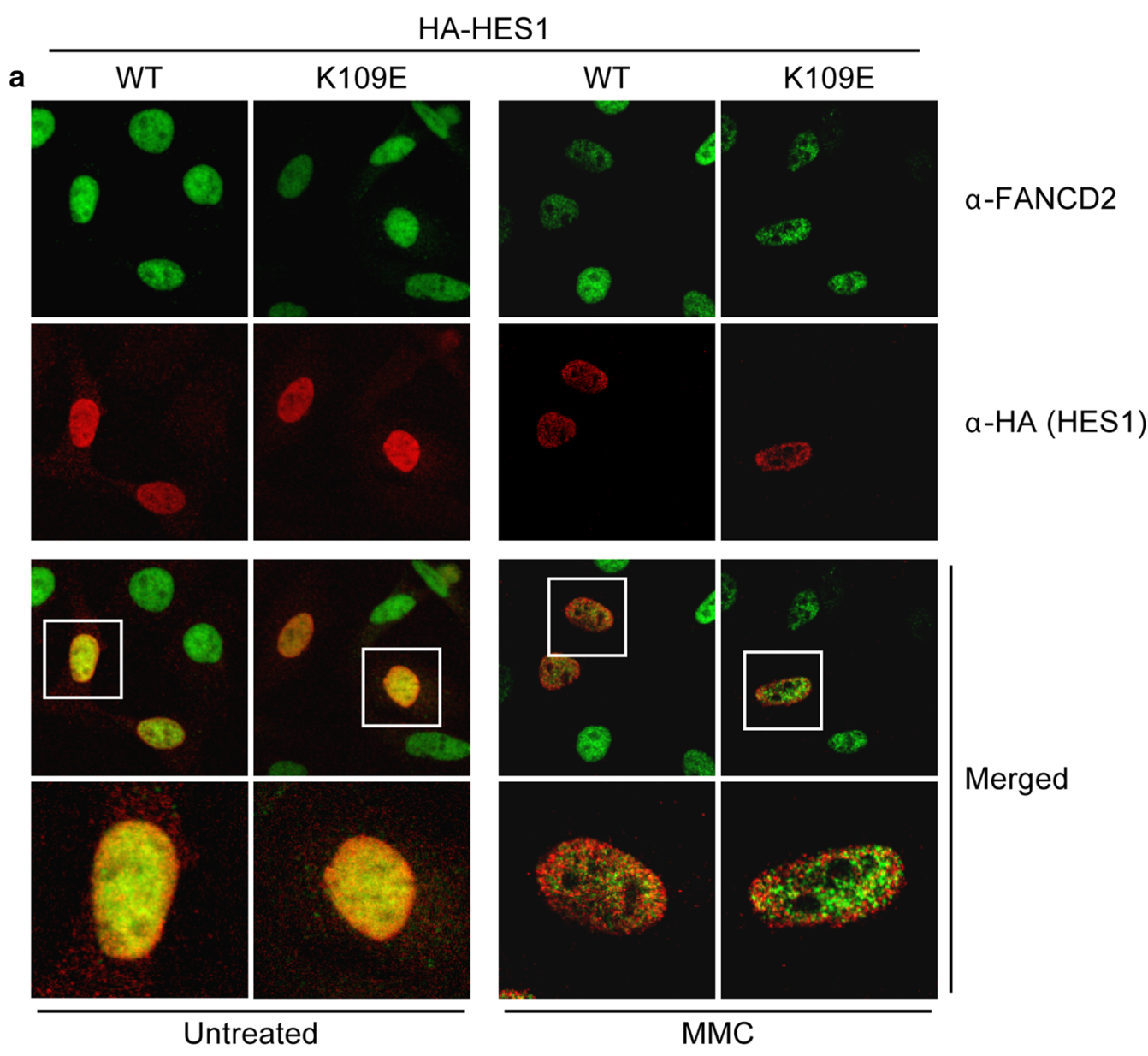

b HES1pro-LUC

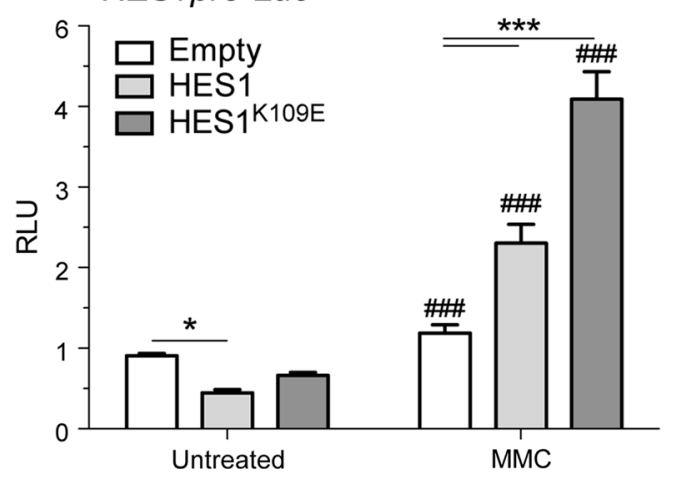

Fig. 2 HES1 mono-ubiquitination is not required for foci formation. a HES1 ${ }^{\mathrm{K} 109 \mathrm{E}}$ mutation does not impair MMC-induced HES1 foci formation. HeLa cells transfected with HA-tagged HES1 or HES1 ${ }^{K 109 E}$ coding vectors were processed for immunofluorescence $16 \mathrm{~h}$ following treatment with MMC (120 ng/ml). Cells were double-stained with anti-HA and anti-FANCD2 antibodies (mouse anti-HA at 1:500; rabbit anti-FANCD2, Novus Biologicals NB100-182 at 1:2000) followed with secondary antibodies (Goat anti-mouse Alexafluor-555, ThermoScientific A-32727 and goat anti-rabbit Alexafluor-488, ThermoScientific, A-11008). Cell nuclei were labelled with TO-PRO-3 lodide stain (1:400, ThermoScientific, T3605). Cells were visualized at 100 $\times$ magnification. b HES1 lysine 109 plays a role in MMC-induced activation of the HES1 promoter. COS-1 cells transfected with pHES1 pro-LUC and HES1 or HES1 ${ }^{\mathrm{K} 109 \mathrm{E}}$ coding vectors $(0.034 \mu \mathrm{g})$ were treated with $120 \mathrm{ng} / \mathrm{ml} \mathrm{MMC}$. Empty vectors were used as controls. Experiments were done twice in triplicates. 2-way ANOVA with Bonferroni correction test, ${ }^{*} p<0.01$ and ${ }^{* * *} p<0.0001$, as compared to Empty; $\# \#$ $<<0.0001$, as compared to Untreated 

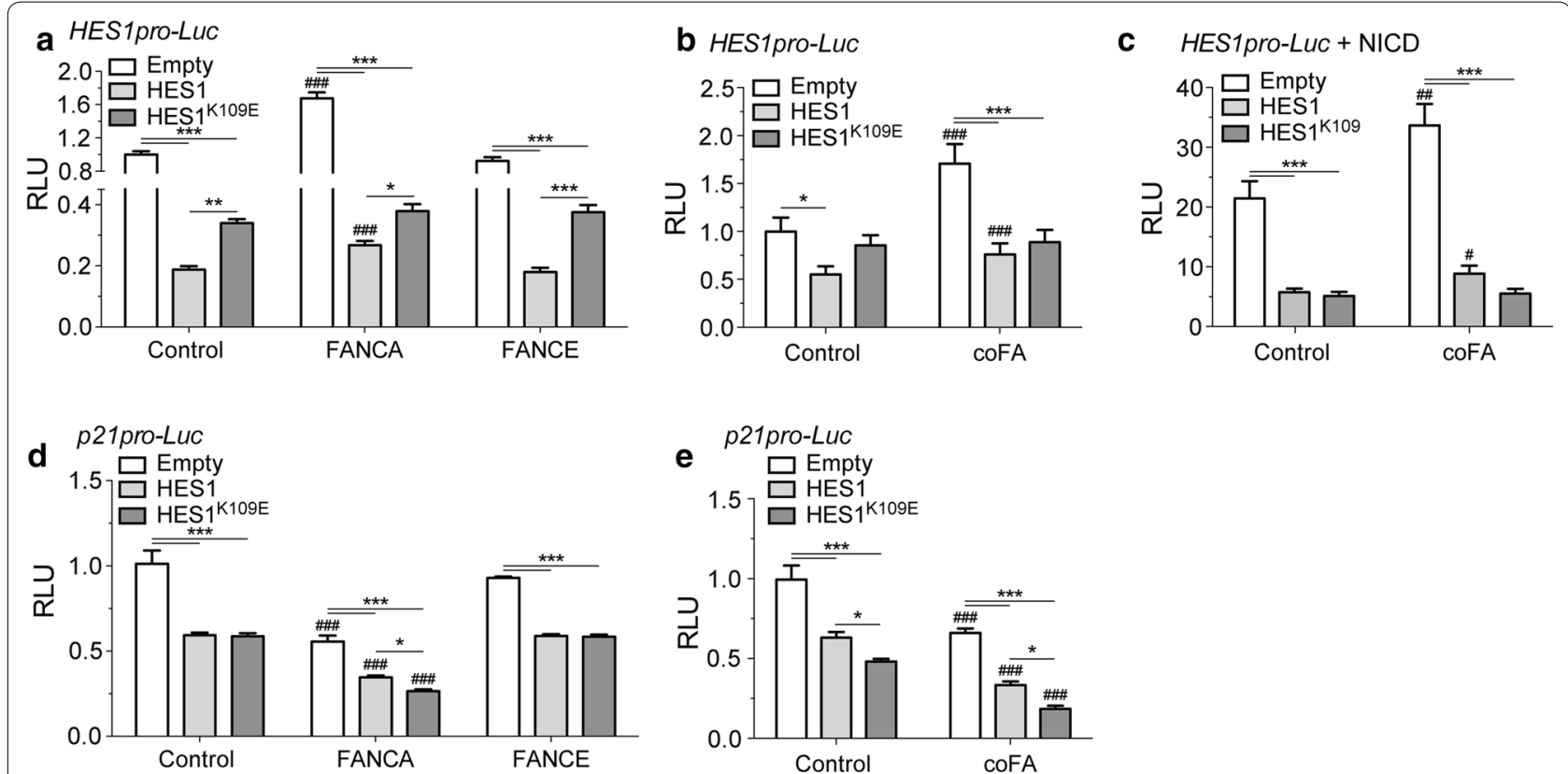

Fig. 3 HES1 lysine 109 is required for HES1 transcriptional regulation. a Altered HES1 expression in FA mutant cells. FA-A cells were transfected with pHES1 pro-LUC and plasmids carrying either the correcting gene FANCA or, as a negative control, FANCE; with the HES1 or the HES1 ${ }^{\text {K109E }}$ expression plasmid. b HES1 lysine 109 is required for FA complex-mediated regulation of HES1. COS-1 cells were transfected with pHES1 pro-LUC and HES1 or HES $1^{K 109 E}$ expression vector with or without plasmids encoding the FA core complex (COFA) and in c with NICD expression plasmid. d Mutation of HES1 lysine 109 promotes FA complex-mediated p21 CIP1MAF1 repression. COS-1 cells were transiently transfected with p21 pro-Luc reporter vector with $\mathrm{HES} 1$ or HES1 ${ }^{\mathrm{K} 109 \mathrm{E}}$ coding vectors, and either with FA complex members coding vectors. e FA complex-mediated repression of $p 21^{\text {CIP1 WAF1 }}$ transcription is enhanced by HES1 K109E mutation. FA-A cells were transiently transfected with p21 pro-Luc and either FANCA or FANCE (negative control) coding vectors, along with wildtype or K109E mutant HES1 coding vectors. All plasmids were transfected at equimolar ratios. Control indicates empty vectors. All experiments were done at least three times in duplicates. 2-way ANOVA with Bonferroni correction test, ${ }^{*} p<0.01$, ${ }^{* *} p<0.005$, ${ }^{* * *} p<0.0001$, as compared to Empty; $p<0.01,{ }^{\# \#} p<0.001$ and ${ }^{\# \# \#} p<0.0001$, as compared to Control

identified as 106 in their publication but identified as 109 in NCBI reference sequence NP_005515.1. This conserved lysine residue is located within a motif similar to the FA recognition sequence found in FANCD2 and FANCI [7-9]. The fact that the same site of HES1 posttranslational modification was identified using different approaches underline the possibility that this conserved residue or protein sequence motif plays a role in HES1 regulation. Indeed, our results suggest that modification of the K109 play a role in HES1 transcriptional regulation. In addition, the structural similarities between the FA core complex and the RBX1-CUL1-SKP1 complex, as proposed by Boisvert and Howlett [16], the direct interaction of HES1 with several members of the core complex [5], and our results presented here suggest that HES1 is a putative target of the FA core complex. Furthermore, we found that $\mathrm{HES}^{\mathrm{K} 109 \mathrm{E}}$ lysine mutant could still interact with members of the FA the core complex (data not shown). Based on our previous findings [6] and results presented here we can hypothesize that monoubiquitination of HES1 influence its affinity to specific promoters and or to other corepressors.
More recently, mutations in the Ring Finger and WD Repeat Domain 3 (RFWD3 or FANCW)-another E3 ligase-was shown to cause FA in a child [17]. Given the complexity of the FA pathway, now composed of two E3 ligases, it is conceivable that other substrates exist. Our results strongly suggest that HES1 is a putative substrate of the FA core complex ubiquitin ligase.

\section{Limitations}

It is unclear whether HES1 mono-ubiquitination described in this work affects its stability and affinity to promoters or binding to corepressors. It is also conceivable that HES1 mono-ubiquitination occurs via other E3 ligases regulated by the FA core complex. Further work is needed to clarify these issues.

\section{Abbreviations}

FA: Fanconi anemia; FA-A: Fanconi anemia group A; FANCA: Fanconi anemia A; FANCD2: Fanconi anemia D2; FANCI: Fanconi anemia I; FANCW: Fanconi anemia W; HA: hemagglutinin; HES1: Hairy Enhancer of Split 1; MMC: mitomycin C; $21^{C \mid P 1 / W A F 1}$ : cyclin-dependent kinase inhibitor 1; RFWD3: Ring Finger and WD Repeat Domain 3; RBX1-CUL1-SKP1: Ring-Box 1-Cullin 1-S Phase Kinase Associated Protein 1. 


\section{Authors' contributions}

CST designed and performed experiments, analyzed data and wrote the manuscript. FFH performed experiments. GL analyzed data and wrote the manuscript. MC designed experiments, analyzed data and wrote the manuscript. All authors read and approved the final manuscript.

\section{Author details}

${ }^{1}$ Australian Centre for Blood Diseases, Monash University, Melbourne, Australia. ${ }^{2}$ Francis Family Liver Clinic, University Health Network, Toronto, ON, Canada. ${ }^{3}$ Department of Psychiatry and Neurosciences, Université Laval, Quebec, QC, Canada. ${ }^{4}$ Department of Pediatrics, Université Laval, CHUL, 2705 Boul. Laurier, RC-9800, Quebec, QC G1V 4G2, Canada.

\section{Acknowledgements}

We wish to thank Dr. Markus Grompe, Oregon Health \& Science University for PD430T cells, Dr. Ron Kopito, Stanford University for ubiquitin vectors (pCW7 and pCW8); Dr. Ryoichiro Kageyama, Kyoto University for the HES1 vector (HES1 pro-Luc), Dr. Claude Labrie, Laval University for the p21 $1^{\text {IP1 } 1 \text { WAF1 }}$ (p21 proLuc) reporter vector.

\section{Competing interests}

The authors declare that they have no competing interests.

\section{Availability of data and materials}

All data generated or analysed during this study are included in this published article.

\section{Consent for publication}

Not applicable.

\section{Ethics approval and consent to participate}

Not applicable.

\section{Funding}

This work was supported by a grant from the Canadian Institutes of Health Research (CIHR) and from the Fondation du CHU de Québec to MC. Additional support was provided by training awards from "La Fondation de la Recherche sur les Maladies Infantiles" and the FROS to C.S.T.

\section{Publisher's Note}

Springer Nature remains neutral with regard to jurisdictional claims in published maps and institutional affiliations.

Received: 11 January 2018 Accepted: 9 February 2018

Published online: 20 February 2018

\section{References}

1. Iso T, Kedes L, Hamamori Y. HES and HERP families: multiple effectors of the Notch signaling pathway. J Cell Physiol. 2003;194(3):237-55.
2. Kageyama R, Ohtsuka T, Kobayashi T. The Hes gene family: repressors and oscillators that orchestrate embryogenesis. Development. 2007:134(7):1243-51.

3. Sang L, Coller HA, Roberts JM. Control of the reversibility of cellular quiescence by the transcriptional repressor HES1. Science. 2008;321(5892):1095-100

4. Castella P, Sawai S, Nakao K, Wagner JA, Caudy M. HES-1 repression of differentiation and proliferation in PC12 cells: role for the helix 3-helix 4 domain in transcription repression. Mol Cell Biol. 2000:20(16):6170-83.

5. Tremblay CS, Huang FF, Habi O, Huard CC, Godin C, Levesque G, Carreau M. HES1 is a novel interactor of the Fanconi anemia core complex. Blood. 2008;112(5):2062-70.

6. Tremblay CS, Huard CC, Huang FF, Habi O, Bourdages V, Levesque $\mathrm{G}$, Carreau M. The Fanconi anemia core complex acts as a transcriptional co-regulator in Hairy enhancer of split 1 signaling. J Biol Chem. 2009;284(20):13384-95.

7. Sims AE, Spiteri E, Sims RJ 3rd, Arita AG, Lach FP, Landers T, Wurm M, Freund $\mathrm{M}$, Neveling $\mathrm{K}$, Hanenberg $\mathrm{H}$, et al. FANCl is a second monoubiquitinated member of the Fanconi anemia pathway. Nat Struct Mol Biol. 2007;14:564.

8. Garcia-Higuera I, Taniguchi T, Ganesan S, Meyn MS, Timmers C, Hejna J, Grompe M, D'Andrea AD. Interaction of the Fanconi anemia proteins and BRCA1 in a common pathway. Mol Cell. 2001;7(2):249-62.

9. Smogorzewska A, Matsuoka S, Vinciguerra P, McDonald ER 3rd, Hurov KE, Luo J, Ballif BA, Gygi SP, Hofmann K, D'Andrea AD, et al. Identification of the $\mathrm{FANCI}$ protein, a monoubiquitinated FANCD2 paralog required for DNA repair. Cell. 2007:129(2):289-301.

10. Meetei AR, de Winter JP, Medhurst AL, Wallisch M, Waisfisz $Q$, van de Vrugt HJ, Oostra AB, Yan Z, Ling C, Bishop CE, et al. A novel ubiquitin ligase is deficient in Fanconi anemia. Nat Genet. 2003;35(2):165-70.

11. Ciechanover A, Schwartz AL. The ubiquitin-proteasome pathway: the complexity and myriad functions of proteins death. Proc Natl Acad Sci USA. 1998:95(6):2727-30

12. Takebayashi K, Sasai Y, Sakai Y, Watanabe T, Nakanishi S, Kageyama R. Structure, chromosomal locus, and promoter analysis of the gene encoding the mouse helix-loop-helix factor HES-1. Negative autoregulation through the multiple N box elements. J Biol Chem. 1994;269(7):5150-6.

13. Shimojo H, Ohtsuka T, Kageyama R. Oscillations in notch signaling regulate maintenance of neural progenitors. Neuron. 2008;58(1):52-64.

14. Kobayashi T, Mizuno H, Imayoshi I, Furusawa C, Shirahige K, Kageyama R. The cyclic gene Hes 1 contributes to diverse differentiation responses of embryonic stem cells. Genes Dev. 2009:23(16):1870-5.

15. Chen F, Zhang C, Wu H, Ma Y, Luo X, Gong X, Jiang F, Gui Y, Zhang H, Lu F. The E3 ubiquitin ligase SCFFBXL14 complex stimulates neuronal differentiation by targeting the Notch signaling factor HES1 for proteolysis. J Biol Chem. 2017;292:20100-12.

16. Boisvert RA, Howlett NG. The Fanconi anemia ID2 complex: dueling saxes at the crossroads. Cell Cycle. 2014;13(19):2999-3015.

17. Knies K, Inano S, Ramirez MJ, Ishiai M, Surralles J, Takata M, Schindler D. Biallelic mutations in the ubiquitin ligase RFWD3 cause Fanconi anemia. $J$ Clin Investig. 2017;127(8):3013-27.

\section{Submit your next manuscript to BioMed Central and we will help you at every step:}

- We accept pre-submission inquiries

- Our selector tool helps you to find the most relevant journal

- We provide round the clock customer support

- Convenient online submission

- Thorough peer review

- Inclusion in PubMed and all major indexing services

- Maximum visibility for your research

Submit your manuscript at www.biomedcentral com/submit
Biomed Central 